\title{
Calcioantagonistas en trabajo de parto pretérmino: Un meta-analisis
}

\author{
Omar Méndez Salamando, ${ }^{*}$ Edinson Caicedo Cerezo, ${ }^{* *}$; Rodrigo Cifuentes***
}

\section{RESUMEN}

OBJETIVO: Revisar los reportes obtenidos en la literatura sobre la eficacia de los calcioantagonistas como drogas uteroinhibidoras en trabajo de parto pretermino.

MATERIALES Y METODOS: Se buscaron estudios clinicos controlados en medline, The cochrane library, internet, Se aplicaron tablas de 2 × 2, se calculó riesgo relativo e intervalo de confianza y se determinó diferencias estadísticamente significantes para tiempo de uteroinhibición y efectos adversos.

RESULTADOS: No hubo diferencias estadísticamente significantes al compararlo con otros tocolíticos en cuanto al tiempo de uteroinhibición. Los calcioantagonistas tuvieron incidiencia menor de efectos adversos siendo estos estadísticamente significantes.

CONCLUSION: Los calcioantagonistas son una buena alternativa como terapia uteroinhibidora en trabajo de parto pretérmino.

PALABRAS CLAVES: Parto pretérmino, calcioantagonistas, ritodrina, sulfato de magnesio.

SUMMARY

OBJETIVE: To revise reports obtained from books about the efficacy of calcioantagonist as a uterus inhibitory drug during premature delivery.

MATERIALS AND METHODS: Controlled clinical studies were searched for in medline the cochrane library, in Internet. $2 \times 2$ tables were applied. Relative risk and trust interval were calulated, and statistically significant differences for uterus - inhibition time and unfavorable effects were determined.

RESULTS: There were not any statistically significant differences when compared with other tocolytic drugs, as for uterus-inhibition time. Calcioantagonist had a minor incidence of unfavorable effects, which were statistically significant.

CONCLUSION: Calcioantogonist are a good alternative as a uterus - inhibitory theraphy when working on premature delivery.

KEY WORDS: Preterm labor, calcioantagonist, ritodrine, Magnesium sulfate.

\section{Introducción}

El parto pretérmino es definido como contracciones uterinas regulares con cambios cervicales progresivos o contracciones uterinas regulares con un cervix dilatado más de $2 \mathrm{cms}$ y borrado en un $80 \%$ antes de la semana 37 de gestación $(3,6)$. Esta entidad es la causa de $80 \%$ de las muertes neonatales no relacionadas con malformaciones congénitas.

Residente de Ginecología 2do. Año Universidad del Valle. Cali Colombia

Estadístico, MSc. en Organizaciones, Profesor Auxiliar de la Universidad del Valle.

Profesor Titular de la Universidad del Valle, Jefe de Sección Medicina Perinatal, Facultad de Salud Univalle.
El parto pretérmino de productos antes de llegar a las 37 semanas de la gestación complica 8 a $10 \%$ de los partos en Estados Unidos y es la causa principal de morbilidad y mortalidad neonatales $(1,5)$.

La prematuridad y el bajo peso al nacer continúan siendo un problema de salud pública (7). La incidencia del bajo peso al nacer en el Hospital Universitario del Valle (Cali, Colombia) en el año de 1.997 fue del 18,1\%; porcentaje considerablemente mayor comparado con la incidencia presentada en estudios publicados en los Estados Unidos, los cuales se mantienen alrededor del $8 \%$ de todos los nacidos vivos. (7)

Uno de los problemas más notables que enfrenta el clínico que trata a una embarazada es corroborar el diagnóstico y emprender el tratamiento del trabajo de parto pretérmino y evitar que nazca un producto antes del término (14). En la solución a este problema, un aspecto 
importante es la utilización de diversos tocolíticos, que incluyen $\mathbf{B}$ simpaticomimeticos, indometacina, magnesio, bloqueadores de vías de calcio y atosibán, un nuevo antagonista de la oxitocina que se encuentra fase III de los estudios.

No existe un tocolítico perfecto y su empleo conlleva algunos problemas posibles para la madre. Todos los agentes solos o en combinación, generan notables efectos adversos.

En el presente artículo se pretende revisar la eficacia de los calcioantagonistas, en cuanto a tiempo de uteroinhibición y efectos adversos con respecto a otros tocolíticos (betamiméticos, sulfato de magnesio,diltiazem) usados en el trabajo de parto pretérmino.

\section{Materiales y Métodos}

Se buscaron artículos en el Centro de Documentación de la Universidad del Valle, Promédico, Fundación Fes a través del Medline, The Cochrane Library, correo electronico internet, publicaciones realizadas en Revistas científicas (Journals) y libros orientados para realizar el diseño de un metaanálisis $(9,10)$

Se seleccionaron varios artículos $(2-6,8,11-12)$ que versaron sobre el uso de calcioantagonistas en trabajo de parto pretérmino y cuyas publicaciones fueron realizadas desde el año 1.986 al año 1998.

La selección de las publicaciones se hicieron basándose en los siguientes criterios:

1. Fueron ensayos clínicos controlados

2. Escritos en inglés, francés, alemán o español

3. El tema fuera calcioantagonistas en trabajo de parto pretérmino y pudiera establecer comparaciones con respecto a otro tocolítico usado en términos de tiempo de uteroinhibición y efectos adversos.

4. Definieran claramente trabajo de parto pretérmino.

5. Tuvieran criterios de inclusión y exclusión similares para la población a estudio: pacientes con trabajo de parto prematuro entre las semanas 20 y 36 de gestación, parto prematuro definido como contracciones regulares acompañadas de cambios cervicales mayor de $2 \mathrm{cms}$ y menor de $4 \mathrm{cms}$ con membranas intactas. Se excluían pacientes con los siguientes factores maternos; exposición tocolítica previa durante el embarazo actual, diabetes, hipertiroidismo, enfermedad cardiaca, preeclampsia, eclampsia, placenta previa, corioamnionitis, gestación múltiple, hidramnios, dilatación cervical mayor que 4 cms, sufrimiento fetal, RCIU, FM ó anomalía fetal incompatible con la vida, constituyeron factores fetales para la exclusión.

6. Las poblaciones aleatorizadas para recibir calcioantagonista u otro tocolítico fueron comparables en cuanto a variables demografica y clínicas y no hubiese diferencias estadísticamente significantes.

7. Todos los estudios considerados posiblemente elegibles fueron revisados independientemente por 2 autores, quienes brindaron asesoría sobre los criterios de inclusión y validez de los estudios. Esto con el fin de disminuir la heterogeneidad entre estudios diferentes.

8. Se estableció la eficacia de los calcioantagonistas, dado en términos de tiempo de uteroinhibicíon y efectos adversos al compararlos con otros tocolíticos.

Para la cuantificación de estas variables (Tiempo de uteroinhibición y efectos adversos) se estableció una tabla de $2 \times 2$, que permitió calcular el riesgo relativo (RR) y mediante las siguiente ecuación se calculó un intervalo de confianza del $95 \%$ que permitió obtener si los resultados arrojados en los diferentes estudios tenían o no significancia estadística

\section{Donde:}

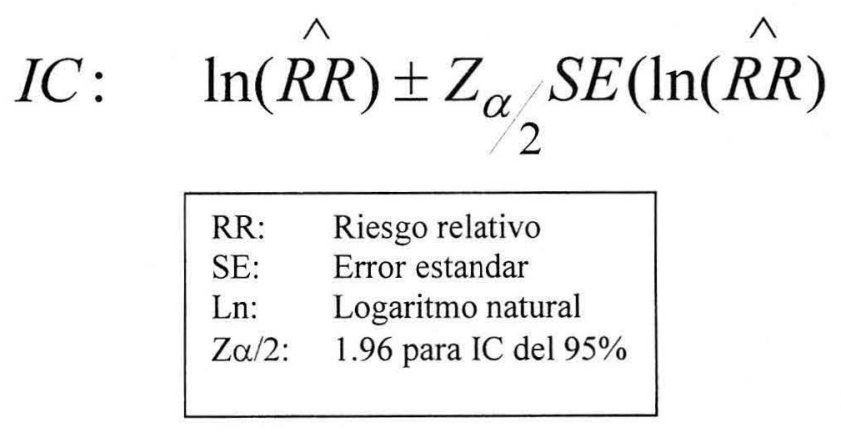

\section{Resultados}

Se revisaron 8 estudios, 6 de los cuales comparaban nifedipina Vs. ritodrina; 1 nifedipina Vs sulfato de magnesio; y otro nifedipina Vs diltiazem.

7 de los 8 estudios (6 nifedipina vs ritodrina, 1 nifedipina vs sulfato de magnesio) permitió evaluar eficacia de uteroinhibición en un tiempo de 48 horas (tabla $1^{\text {a }}$ y tabla $1 b)$.

Tabla 1 a

ESTUDIO DE LA EFICACIA DE LA NIFEDIPINA VS. RITRODINA

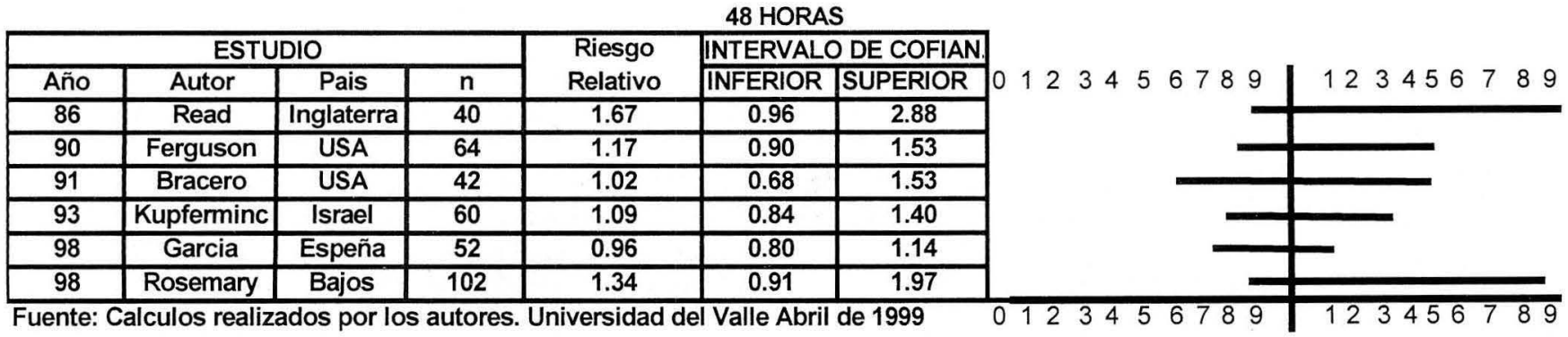


Tabla 1b

ESTUDIO DE LA EFICACIA DE LA NIFEDIPINA VS. SULFATO DE MAGNESIO

\begin{tabular}{|c|c|c|c|c|c|c|c|c|}
\hline \multicolumn{9}{|c|}{ 48 HORAS } \\
\hline \multicolumn{4}{|c|}{ ESTUDIO } & \multirow{2}{*}{$\begin{array}{c}\text { Riesgo } \\
\text { Relativo }\end{array}$} & \multicolumn{2}{|c|}{ INTERVALO DE COFIAN } & $\begin{array}{llllllllll}0 & 1 & 2 & 3 & 4 & 5 & 6 & 7 & 8\end{array}$ & 123456789 \\
\hline Año & Autor & Pais & $\mathbf{n}$ & & INFERIOR & SUPERIOR & & \\
\hline 93 & Glock & USA & 80 & 1.00 & 0.88 & 1.13 & & \\
\hline
\end{tabular}

3 de los 8 estudios (nifedipina vs ritodrina) permitió comparar eficacia de uteroinhibición en un tiempo de 7 días (tabla 2).

3 de los 8 estudios (nifedipina vs ritodrina) permitió comparar eficacia de uteroinhibición a las 36 semanas de gestación (tabla 3).

con respecto al estudio de nifedipina vs diltiazem no permitió realizar iguales comparaciones, debido a que las dos drogas fueron utilizadas en poblaciones que inicalmente se les dio tratamiento con sulfato de magnesio como tocolisis de emergencia, y el diseño del estudio estaba encaminado a medir efectos como terapia de mantenimiento en parto pretérmino después de utilizar una droga de emergencia.

Al comparar la nifedipina con la ritodrina y sulfato de magnesio no hubo diferencias estadísticamente significantes en el tiempo de uteroinhibición (48 horas, 7 días, 36 semanas). Pero con respecto a efectos adversos estas diferencias fueron significantes estadísticamente a excepción del estudio que comparaba nifedipina vs sulfato de magnesio (tabla 4a y tabla 4b).

En el estudio de nifedipina vs diltiazem se pudo establecer que menos pacientes en el grupo de diltiazem comparado con el grupo de nifedipina lograron llegar a las 37 semanas. Hubo más días ganados en utero en este último grupo. La necesidad de reiniciar sulfato de magnesio por fracaso en el mantenimiento de la uteroinhibición en ambos grupos, la incidencia fue similar, siendo estadísticamente no significante (tabla $5 \mathrm{a}$ y tabla $5 \mathrm{~b}$ ).

\section{Discusion}

Los bloqueadores de vías de calcio (ó antagonistas de calcio), constituyen un grupo heterogeneo de compuestos que actúan fundamentalmente al inhibir la penetración de los iones calcio por la membrana celular. El grado de activación de las proteínas contractiles de músculo liso

Tabla 2

ESTUDIO DE LA EFICACIA DE LA NIFEDIPINA VS. RITODRINA

7 DIAS

\begin{tabular}{|c|c|c|c|c|c|c|c|c|}
\hline \multicolumn{4}{|c|}{ ESTUDIO } & \multirow{2}{*}{$\begin{array}{l}\text { Riesgo } \\
\text { Relativo }\end{array}$} & \multicolumn{2}{|c|}{ INTERALO DE COFAN } & \multirow[t]{2}{*}{01233456789} & \multirow[t]{2}{*}{123456789} \\
\hline Año & Autor & Pais & $\mathrm{n}$ & & INFEROR & SUPETOR & & \\
\hline 90 & Ferguson & USA & 64 & 1.10 & 0.74 & 1.64 & & \\
\hline 93 & Kupferminc & Israel & 60 & 1.05 & 0.73 & 1.53 & & \\
\hline 98 & Rosemary & Bajos & 102 & 1.06 & 0.69 & 1.62 & & \\
\hline
\end{tabular}

Tabla 3

ESTUDIO DE LA EFICACIA DE LA NIFEDIPINA VS. RITODRINA

36 SEMANAS

\begin{tabular}{|c|c|c|c|c|c|c|c|c|}
\hline \multicolumn{4}{|c|}{ ESTUDIO } & \multirow{2}{*}{$\begin{array}{l}\text { Riesgo } \\
\text { Relativo }\end{array}$} & \multicolumn{2}{|c|}{ INIERVALODECOFAN } & \multirow{2}{*}{ ] 0123456789} & \multirow[t]{2}{*}{123456789} \\
\hline Año & Autor & Pais & $\mathbf{n}$ & & INFEAOR & SUPEROR & & \\
\hline 90 & Ferguson & USA & 64 & 0.79 & 0.42 & 1.47 & & \\
\hline 93 & Kupferminc & Israel & 60 & 1.15 & 0.67 & 1.99 & & \\
\hline 98 & Rosemary & Bejos & 102 & 1.63 & 0.88 & 3.02 & & \\
\hline
\end{tabular}


Tabla 4 a

ESTUDIO DE EFECTOS ADVERSOS DE LA NIFEDIPINA VS. RITODRINA

\begin{tabular}{|c|c|c|c|c|c|c|}
\hline \multicolumn{4}{|c|}{ ESTUDO } & & \multicolumn{2}{c|}{ INIERVALO DECOFAN } \\
\hline Año & Autor & Pais & $\mathrm{n}$ & Riesgo Relativo & INFEROR & SUPEROR \\
\hline 86 & Read & Inglaterra & $\mathbf{4 0}$ & 0.15 & 0.04 & 0.60 \\
\hline 90 & Ferguson & USA & 64 & 0.28 & 0.11 & 0.67 \\
\hline 91 & Bracero & USA & 42 & 0.34 & 0.15 & 0.80 \\
\hline 93 & Kupferminc & Israel & 60 & 0.35 & 0.19 & 0.65 \\
\hline 98 & Garcia & Espeña & 52 & 0.17 & 0.06 & 0.50 \\
\hline
\end{tabular}

Fuente: Calculos realizados por los autores. Universidad del Valle Abril de 1999

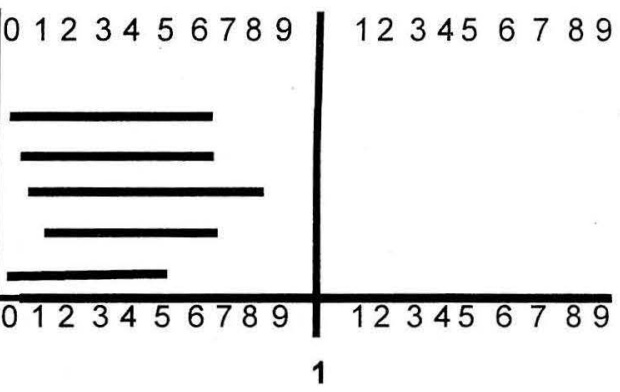

Tabla 4b

ESTUDIO DE EFECTOS ADVERSOS DE LA NIFEDIPINA VS. SULFATO DE MAGNESIO

\begin{tabular}{|c|c|c|c|c|c|c|c|c|}
\hline \multicolumn{4}{|c|}{ ESTUDOO } & \multirow[b]{2}{*}{ Riesgo Relativo } & \multicolumn{2}{|c|}{ INIGRALODECOAAN } & \multirow{2}{*}{0123456789} & \multirow[t]{2}{*}{123456789} \\
\hline Año & Autor & Pais & $\mathbf{n}$ & & INFEAR & SUPEROR & & \\
\hline 93 & Gock & USA & 80 & 0.40 & 0.16 & 1.03 & & \\
\hline Fuent & Jallos & dos & $\ln$ & itores Univer & del Valle Ab & 99 & 012345678.9 & 12345678 \\
\hline
\end{tabular}

Tabla 5a

ESTUDIO DE LA EFICACIA DE LA NIFEDIPINA VS. DILTIAZEM

MAYOR DE 37 SEMANAS

\begin{tabular}{|c|c|c|c|c|c|c|c|c|}
\hline \multicolumn{4}{|c|}{ ESTUDIO } & \multirow{2}{*}{$\begin{array}{l}\text { Riesgo } \\
\text { Relativo }\end{array}$} & \multicolumn{3}{|c|}{ INTERVALODE COFIAN] 01233456789} & \multirow[t]{2}{*}{123456789} \\
\hline Año & Autor & Pais & $\mathbf{n}$ & & INFEROR & SSUPEROR & & \\
\hline 98 & Yasser & $\overline{\text { USA }}$ & 69 & 2.75 & 1.12 & 6.73 & & \\
\hline
\end{tabular}

Tabla 5b

ESTUDIO DE LA EFICACIA DE LA NIFEDIPINA VS. DILTIAZEM RETOCOLISIS CON SULFATO DE MAGNESIO

\begin{tabular}{|c|c|c|c|c|c|c|c|c|}
\hline \multicolumn{4}{|c|}{ ESTUDIO } & \multirow{2}{*}{$\begin{array}{l}\text { Riesgo } \\
\text { Relativo }\end{array}$} & \multicolumn{3}{|c|}{ INTERVALODE COFIAN, 01234566789} & \multirow[t]{2}{*}{123456789} \\
\hline Año & Autor & Pais & $\mathbf{n}$ & & INFERIOR & SUPEROR & & \\
\hline 98 & Yasser & USA & 69 & 0.79 & 0.43 & 1.45 & & \\
\hline
\end{tabular}

guarda relación directa con los cambios en la concentración del calcio citoplasmático. La concentración del calcio libre en el citoplasma puede aumentar por la penetración de dicho mineral a través de las vías (conductos) que dependen de voltaje o son operados por receptores.

El calcio también puede ser liberado de los sitios de unión en el interior de la célula como sería la superficie interna de la membrana celular y también el retículo sarcoplásmico y las mitocondrias. El incremento en el calcio libre ocasiona la unión del mineral a la calmodulina, que más adelante se fosforila y activa la cinasa de cadena ligera de miosina, que a su vez activa la miosina y ocasiona su contracción. El sitio principal de acción de los bloqueadores de vías de calcio, según algunos investigadores, son los conductos que dependen de voltaje, en los que la inhibición de la penetración del calcio extracelular ocasiona desacoplamiento de la excitación de la contracción. La inhibición de la penetración de dicho mineral, además de disminuir de manera directa la concentración en que está en el citoplasma, también puede causar un decremento en la liberación del calcio de reservas intracelulares. Así mismo, se han sugerido otros efectos relajantes de dichos fármacos en etapas ulteriores del proceso de acoplamiento de excitación/contracción.

Existen diversos tipos de bloqueadores de vías de calcio que se clasifican con base en su estructura química 
central; todos inhiben la penetración de calcio, pero tienen una gran diversidad de acciones. El verapamilo es un bloqueador de tipo papaverinico que puede relajar el miometrio in vitro. Sin embargo, también muestra efectos inhibidores en la conducción del nodo auriculoventricular y en la contracción de músculos cardiaco y liso. Los efectos del verapamilo en el corazón, que incluyen más bien merma de la conducción auriculo ventricular, limitan en grado significativo la dosis tolerable, y se ha demostrado que no es eficaz para combatir el trabajo de parto pretérmino. Algunos investigadores han recomendado agregar verapamilo a los $\mathbf{B}$ mimeticos para llevar al mínimo los efectos adversos en el árbol cardiovascular que son propios del uso de éstos últimos. Sin embargo en estudios de laboratorio y clínicos no se ha demostrado efecto cardio protector alguno del verapamilo. Los bloqueadores de vías de calcio del tipo de la 1,4 dihidropiridina, como son la nifedipina y la nicardipina, son inhibidores más selectivos de las contracciones uterinas. Los efectos que ellos tienen en la conducción auriculoventricular son menos intensos en el caso de la nifedipina, y no se observan con las dosis utilizadas en seres humanos. La nifedipina es el bloqueador prototipo de vías de calcio que se utiliza en investigaciones clínicas como tocolítico. (13)

Los estudios revisados permitieron establecer comparaciones en el tiempo logrado de uteroinhibición siendo demostrado que la nifedipina tiene similar eficacia que las otras drogas tocolíticas (ritodrina, sulfato de magnesio) y no hubo diferencias estadísticamente significante a las 48 horas, 7 días, 36 semanas de uteroinhibición.

Al comparar los efectos adversos es claro que la nifedipina tiene una menor incidencia de efectos adversos al compararla con los otros tocolíticos (ritodrina) siendo estos resultados estadísticamente significantes.

En el caso particular del estudio con sulfato de magnesio hubo menos incidencia de efectos adversos en el grupo de nifedipina al compararlo con el grupo de sulfato de magnesio. Pero estas diferencias no fueron estadisticamente significantes. Aunque en el grupo que recibió sulfato de magnesio los efectos adversos fueron de mayor gravedad, hasta el punto de tener que suspender la medicación en algunas de las pacientes (6).

Los efectos adversos en el grupo de sulfato de magnesio más frecuentes fueron dolor toracico, edema pulmonar, hipotensión transitoria. Los efectos más indeseables en el grupo que recibió nifedipina fueron cefalea, hiperemia facial y nausea. (6)

En el grupo que recibió ritodrina (beta-simpaticomimético) los efectos adversos más frecuentes fueron taquicardia, falta de aire, dolor retroesternal, nausea, hiperglicemia, etc. (14)

El estudio de nifedipina vs diltiazem se distribuyeron al azar dos grupos después de una tocolisis inicial con sulfato de magnesio, en pacientes con trabajo de parto pretérmino. Se comparó la eficacia de nifedipina como droga de mantenimiento o sosten después de una tocolisis de emergencia.

Hubo una mayor proporción de pacientes en el grupo de nifedipina que alcanzaron parto después de las $37 \mathrm{ss,}$ mayores días ganados en útero y mayor edad gestacional al momento del parto. Datos estadísticamente significantes.

Aunque ha sido una practica dar terapia de mantenimiento en pacientes con trabajo de parto pretérmino, el debate continúa sobre la efectividad de esta conducta en los reportes de la literatura mundial. Algunos autores no reportan beneficios en la prolongación del embarazo ó en la reducción de la recurrencia del parto prematuro con terapia de mantenimiento oral comparada con placebo. $(15,16)$. Otros investigadores han sugerido una ventaja significante con la terapia de mantenimiento $(17,18)$. Estos estudios se han realizado con terapia de mantenimiento utilizando beta-miméticos o magnesio oral vs placebo.

Los bloqueadores de canales de calcio como es sabido no inducen taquifilaxia y en este sentido podrían ser más efectivos que los beta-miméticos o placebo para realizar tocolisis de mantenimiento. Se necesitarían realizar estudios para dar respuesta a este interrogante.

Por lo anteriormente expuesto se puede concluir que los calcioantagonistas son una buena alternativa en el tratamiento de parto pretérmino comparada con los otros tocolíticos. Estas cualidades la podrían convertir como droga de primera línea en la prevención de trabajo de parto pretérmino. Esta conclusión sería válida si se descarta un problema importante del meta-análisis, que es la predisposición de la publicación (sesgo de publicación). Esto ocurre cuando los estudios que no tienen resultados significantes ó tienen resultados negativos son publicados con menos frecuencias que los estudios positivos.

\section{BIBLIOGRAFIA}

1. Roberts JM. Current understanding of pharmocologic mechanismis in the preventión of preterm birth. Clin Obstet Gynecol. 1988, 27: 52-605.

2. Read MD, Wellby DE. The use of a calcium antagonist (nifedipine) to suppress preterm labour. Br Obstet Gynaecol. 1986; 93: 933-937.

3. Ferguson JE, Dyson DC, Schutz T, Steven son DK,Acomparison of tocolysis with nifedipine or ritodrine: analysis of efficacy and maternal fetal and neonatal outcome. Am J Obstet Gynecol 1990;163:105-111.

4. Bracero LA, LEIkin E, Kirshenbaum N, Tejani N. Comparison of nifedipine and ritodrine for the treatment of preterm labor Am J, Perinatol.1991; 8:365-369.

5. Kupterminc M. Lessing JB, Yaron Y, peyser MR, Nifedipine Versus ritodrine for suppresson of preterm labour. $\mathrm{Br}$ Obstet Gyneacol 1993;100:1090-1094
6. Glock Jacob L., Morales Walther J., efficacy an safety of nifedipine versus magnesium sulfate in the management of preterm labor: A randomized study AM J. Obstet Gynecol 1993; 169: 960-963.

7. Cifuentes Rodrigo, Leon Jorge, Trochez Luz Maria, Estudio comparativo entre Nifedipina - Terbutalina en el trabajo de parto pretermin Revist Colomb de Obstetric y Ginecol. 1994; 45: 117- 121.

8. Velazco Garcia JA. -, Gonzalez Gonzalez A. a prospective, Randomized trial of nifedipine VS ritodrine in Threatened preterm labor. International Journal of Gynecol and Obstetric 1998; 61: 239-244.

9. Bletter María, Sauerbrei Willi. Traditional Reviews, meta analysis and Pooled analyses and Epidemiology. Intern jurnal of Epidemiology 1999; 28: 1-9.

10. Rothman Kenneth, Greeland Sander. Modern Epidemiology Secound Edition. California Hippinneott Raven Pubblishers 1998 
11. Yassery Elsayed, Harold Holbrook, Ronald Gibson. Diltiazem for maintenance tocolysis of preterm labor: caomparison to nifedipine in a randomized trial. The Journal of matern-fetal medicine. 1998; 7: 217-221.

12. Rosemary Frei. Heart Drug may be preferable for preventing preterm labor. European Journal of obstetrics y gynecology and reproduct biology. 1998; 77: 171-176.

13. Ray Deborah, Dyson Donald. Bloqueadores de vías de calcio. Clinicas obstetrics de nort 1995; 4:685-691.

14. Hill Clark Washington. Riesgos y complicacioes de la tocolisis. Clinics Obstetrics de Nort 1.995; 41: 697-709

15. Ricci JM, Seemanthini H, Helfgott A, Reed K, O'Sullivan MJ: Oral tocolysis with magnesium chloride: A randomized controlled prospective clinical trial. Am J Obstet Gynecol 1991; 165: $603-610$.

16. Ruts O, Bofill J, Arriola R, Andrew M, Morrison J: The clinical efficacy oral tocolytic therapy. Am, Obstet Gynecol 1996; 175:838-842.

17. Cresay RK, Golbus MS, Laros RK, parer J, Roberts: J: Oral ritodrine maintenance in treatment of preterm labor: Am J Obstet Gynecol 1980; 137:212-216

18. Lewis R, Mercer BM, Salama M, Walsh MA, Sibai BM: Oral terbulatine after parental tocolysis: A randomized, double, blind, placebo, controlled trial. Am J Obstet Gynecol 1996; 175: 834: 837 . 